In doing a post.mortem examination of this kind, one naturally looks first for the commoner causes of acute disease, and the appendix is early searched for on this account. Unfortunately, the moving of the intestines in the search for the appendix disturbed the position of the ileum, and the position of the upper limit of the volvulus must remain more or less hypothetical. The extreme distension affected about $20 \mathrm{in}$. of the ileum, and the upper limit of the volvulus was probably in this position. In recorded cases of volvulus of the ileum, both the upper and lower limits seem in some cases to have been more or less indefinite.

Death supervened in five hours in this case, and was undoubtedly due to shock following an acute strangulation of a comparatively large amount of bowel, and that bowel part of the small intestine which is of much more vital importance than the large. From the immense size this part of intestine reached, and the amount of congested blood in it, there was practically a severe haemorrhage into it, as far as the general circulation was concerned. This must have greatly increased the already severe shock from which the patient was suffering.

Shock is defined as a condition of exhaustion of the vasomotor centre. Crile has shown that if the abdomen of an animal is opened and the intestines exposed, a fall of blood pressure occurs. This is associated with a vascular dilatation of the splanchnic area, and a marked degree of shock occurs. The animal bleeds into its own portal system. Anaemia of the vasomotor centre results, and sets up a general vasomotor paralysis, which increases as the vasomotor centre becomes exhausted. Treves states that " the grave initial symptoms of intestinal obstruction do not depend upon the fact that the bowel is obstructed, but upon the severe injury to the sympathetic nerves involved." Crile's observations show that this injury acts by producing an inhibition of the vaso-constrictor nerves, and ultimate paralysis of these nerves by exhaustion of the vasomotor centre. The resulting dilatation of the vessels of the splanchnic area causes a great fall in the systemic blood pressure and coincident shock.

In the case of this man there was an added haemorrhage into the part of the intestine forming the volvulus, thus increasing the factors causing shock, and making it much more profound owing to the added anaemia of the brain.

I can find no record of death ensuing in so short a time from intestinal obstruction, although several cases have been recorded where death ensued within twenty-four hours. Dr. Bryant suggests that the man was one who did not complain much, and that he may have had some pain earlier than the time stated.

The part of the ileum involved in this case, the lower part, is most frequently the seat of volvulus when it occurs in the small intestine. Treves says "the majority of patients exhibiting this form of volvulus have been males with a mean age of 25 years." Cases of volvulus of the small intestine in cases of typhoid fever, simulating perforation, are mentioned by Roberts; the symptoms were severe abdominal pain and collapse. Eliot has recorded a case in which a volvulus of the ileum, associated with one of the pelvic colon, caused great shock, with a pulse of 140 , thready and very weak, coldness and lividity, and apathy, but the facial expression showed intense suffering. In the case I have recorded the symptoms of shock were present to such a degree as to cause death within a few hours.

\section{TWO CASES OF APPENDICITIS.}

\section{By H. GOODWYN, F.R.C.S.EDIN.,} BOVEY TRACEY.

The following two cases of appendicitis presenting some unusual features are, I think, worth recording.

$$
\text { CASE I. }
$$

Mrs. C., aged 36, had for a period of two months repeated attacks of severe appendicular colic, accompanied on each occasion by a rise of temperature- $101^{\circ}$ to $102^{\circ}$-with severe abdominal pain, referred at first to the umbilical region, and latterly localized to the region of the appendix, the maximum point of tenderness being over McBurney's point. These attacks subsided under hot fomentations, rest in bed, etc., but the attacks became more frequent and intervals shorter; also they were more severe in character. The patient was three months pregnant, but in the light of the increasing severity of the pain, and frequency of attacks, it was decided to operate, and at the worst the risk of miscarriage was less than the possibility of the formation of an abscess.

Operation.-The caecum was much bound down by adhesions, and the tip of the appendix was adherent to the pelvic brim. There was great difficulty in withdrawing the caecum sufficiently to expose the base of the appendix, and to free the appendix itself, owing to numerous adhesions, but eventually this was accomplished, and a long inflamed appendix was removed, and the stump invaginated by purse-string suture, and covered by Lembert's sutures.

The subsequent history was uneventful; on the next day temperature and pulse were normal, and on the fourteenth day the patient returned home; no signs of uterine disturbance had occurred, and she remained well after six weeks. On opening the appendix, a nematode worm about $1 \frac{1}{2}$ in. in length was found, and there was a haemorrhagic condition of the mucous. lining of the whole organ, and an ulcer commencing to form on the floor, which would in all probability have gone on very shortly to perforation. I think the result amply justified the possible risk of cutting short the pregnancy.

\section{CASE II.}

A child aged 4, who for about twelve months had had attacks nome very severe, of abdominal pain without localized tenderness. principally referred to the umbilical region. Six weeks before operation she had a severe attack of pain, with rise of temperature to $102^{\circ}$; vomiting and marked constipation. There was no tenderness over the appendix at first; in about four days the pain was localized. The appendix, which was much inflamed, $4 \frac{1}{3}$ in. long, and considerably swollen, was isolated without difficulty. It was treated by invagination with purse-string suture, and closed over with Lembert's sutures. The recovery was uneventful; there was no rise of temperature or pulse, and the stitches were removed on the eighth day.

On opening the appendix a haemorrhagic condition of the mucous lining was seen, and a small fishbone, about ${ }_{5}^{5}$ in. long. lying free in the lumen. There was also evidence of an old kink, as represented by a transverse band of scar tissue.

Both these cases are illustrative of the advantage of early operation when symptoms of colic recur without. obvious cause; in the second case, though the temperature: and pulse became normal in a few days, during the whole five weeks there was never complete freedom from slight. attacks of pain, sometimes lasting only a few minutes, norfrom tenderness on deep pressure over the appendix.

The technique I practised was by incision through the skin over the outer border of the rectus, which was pushed inwards without splitting its fibres. 1 think this method" has distinct advantages over the gridiron method, as especially in the first case it would have been nearly impossible to obtain sufficient room for the necessary manipulations without dividing the fibres of the internab oblique and transversalis. I followed Professor von Eiselsberg, of Vienna, in painting the skin with a spirit. lotion of iodine liniment both before the incision and after the stitches were introduced.

\section{A CASE OF VERY LARGE GALL BLADDER. SLCCESSFULLY TREATED BY EXCISION.*}

By F. W. COLLINSON, M.D.Edin., M.R.C.P.LOND.\% F.R.C.S.EDIN.

HONORARY MEDICAL:OFFICER, PRESTON ROYAL INFIRMARY.

THIs case is remariable owing to the immense size of the gall bladder, being amongst the largest on record.

Mrs. S., aged 31, was sent to me by Dr. Callanan of Longridge on October 14th, 1908, suffering from general abdominas distension and pain in the right side of the abdomen.

\section{History.}

There was nothing of note in the family history, as all were healthy; the mother had died in childbirth. The patient herself had always been in good health, though pale. She had two. children, now living and healthy, born six and three years ago, with one miscarriage in between them.

The history of the present affection appeared to date from August, 1907, when she had a fall, for soon after she began to vomit each day, and in December she remained in bed on that account. She distinctly said there was then no swelling of the abdomen. In January she observed a swelling of the lower part. of the abdomen towards the right side. On February 13th she: had greatly increased in size, and was somewhat emaciated and markedly jaundiced. She was tapped, and 25 pints of fluid was drawn off, and the fluid must have been similar to that removed afterwards at the operation. Dr. Callanan was able to "feel the liver for 2 in. or 3 in. below the ribs," but he also wrote, rather prophetically, "this may, of course, be the sac of

Notes read, and patient shown, to the Clinical Branch of the Preston Medico-Ethical Society, February 3rd, 1909 
a cyst." It might have been the cyst coutracted, but it was more likely to be the liver as the cyst would tend to fall away from the liver in all probability, for there would not be any adhesions at the first tapping, as they would arise from oo\%ing after the puncture. Her general health improved, and the jaundice diminished. She, however, began to refill, and on April 10th 25 pints were again drawn off. Her general health still further improved, and her menstruation, which had ceased in the December, recommenced in June, and was regular ul to the operation.

\section{Condition qhen First Sien.}

Upon examination there was no cachexia or jauvdice and her v)ulse and tomperature were normal. Fixcenting the evident enlargement of the abdomen there did not appear to be much the matter with her. The circumference at the umbilicus was 70 in., although she is short in stature. 'The abdomen lid not appear irregular to touch or appearance; it was yuite uniformly dull all over, as I failed to find a tympanitic area anywhere. It did not feel tight, but soft and fluctuating. and a thrill was readily propagated in any part. On vaginal examination the aterus was found to be normally placed, and there was not any bulging in the fornices. The diagnosis was not by any means clear. but, as lincher says, "the conspicuous bulk of the growth at once tnins the thoughts of the observer to the commonest form of abdominal tumour, ovarian crst." I thought it most form of abdominal tumour, ovarian cyst. I thought it most inaty to be an ovarian cyst, but could not be certam, and, as a

inatter of fact,

The same day she was admitted into Preston Infirmary. The arine was 1028, acid, no albumen, no sugar, urates, and no marked coloration of bile, though it must be confessed we dicl not think of testing for bile, as we dicl not get the history of inundice till afterwards from Dr. Callanan.

$$
\text { Operation. }
$$

On October 19th Dr. R. M. Glover gave chloroform, but juickiy changed to ether by the open methorl, which he continued throughont, and with the assistance of Dr. $\mathrm{A}$. Toulmin I operated, the incision being in the midale line below the operated, the incision being in the minas the lavers of periImbilicus. It was impossible to make out the layers of periconcum and sac owing to the very frm adhesion, and the a large trocar, and 20 pints of thick ramboge-coloured liquid a large trocar, and 20 pints of thick gnmboge-coloured liquid escaped, leaving 2 pints at least in the bottom of the crst. Con opening up the sac, the velvety appenrance of the inmer lining was remarkable, reminding us of miniature valvulae comniventes covering the whole surface.

Commencins to separate the cyst, we found that it mennt really tearing the sac from its attachments to the entirenterior ablominal wall, the bladler, the spleen, the stomach. and the liver. Fortuately it was not anvwhere attached to the intesfines, or the firmness of the adhesions would have renclered removal quite impossible: the intestines were all placed quite behind the cyst, which formel a complete cover to them. IIore behind the cyst, which formel a complete cover to them. Nore fluid continued to well up into the cyst, and was seen to bc comin:s out of the dilated cystic duct. There were not any calculi to be found. Another sinus ran up towards the right kicluey, but it proved to be a cul-de-sac formed possibly by the pushing of the cyst upwarls into that region. When the cyst was separated up to the under surface of the liver, the patient became so exceedingly collapsed, from the length of the operation (about two hours) and the violence required in the separation of the adhesions, that the anaesthetist asked for the operation to be brought to a sneedy conclusion : hebce careful examination of the common duct and helatic duct was impos sible, and we had to be content with cutting off the sac and ligaturing drainage tubes into the crstic duct and the afore. mentioned cul-de-sac, and rapidly stitching up the abilomen.

The collapse was gleat, but with the ail of hypodermic and rectal saline injections the paticnt gradually improved, and in forty-eight hours the pulse was 80 and of gool tension. The temperature during the first week never rose alove 100 , and was usually normal. The fluid freely drainet away lye the tube in the crstic duct, the amount being in the first six days respectively $8 \mathrm{oz} ., 4 \mathrm{oz} ., 10 \mathrm{o \%}, 11 \mathrm{oz} ., 16 \mathrm{oz}$., $12 \mathrm{oz}$., and of $a$ similar character to that, removed during the operation. Cpon iressiug the wound on the sixth day all was perfectly healed. excepting where the tubes passed. The fluid continued to escape until November $2 n d$, when the tubes were removell, the quantities having been $14 \mathrm{oz} ., 18 \mathrm{o} \%,, 20 \mathrm{oz}, 16$ o\%., $15 \mathrm{o} \%, 6 \mathrm{o} \%$, $120 \%$. The temperature immediately rose to $1 \mathrm{C2}$, and remained :so till the tube was replaced, when it became normal. The tube was removed altogether on December 20th, when the amount of fluid in twenty-four hours wis from $2 \% \%$ to $40 \%$, and it had changed to a yellowish purulent fluid. There was never any bile in her urine after the operation; the facces were very pale at first, but gradually decpened in colour. She left the infumary on .January 3rd, the sinus being dressel once in twents-four hours, and discharging about $10 \%$. The skin throughout the whole illness was never irritated br the bile When last seen, on March 20th, she complained of occasiona pain in the region of the liver, and her moutl temperature was 99.2 . She described her health as good, and said she was quite able to do her housework. The faeces have been for some time coffee coloured. She is pale, but, as stated before, has alwars been so. There was only slight moistening of the small pad covering the sinus.

Specimens of the fluid and of the cyst wall were sent immediately after the operation to my friend, F. IT. Eurich, M.D.Edin., Honorary Physician and Honorary
Pathologist to Bradford Infirmary, and he kindly reported as follows :

The cyst wall is composed of dense fibrous tissue, the fibres arranged parallel to each other. Its outer half is poor in cell arranged parallel to each other. Its, showing hyperplasia and elements. Its inner hall multiplication of the fixed tissue cells, and also small roundcell (leucocyte) inflitration. These cell massea are developed at fairly regular intervals, so that the inner sulface of the cyst wall is thrown into folds. The inner surface is covered by a thin layer of necrotic tissue in which no structure is recoynizable. No epithelial elements are visible. A grain of bile of the crst separate, on standing, into three layers. The of the crst separate, on stan fat, the middle of a dense albuminous licpuid, bile-stained; the lowest of a granular deposit of bile pigment. Chemically the fluid is rich in albumen, fat, bile pigment, and bile acids. There is no cholesterin. laintly alkaline. Microscopically fat slobules and bile pigment, \& few isolated leucocytes, and one or two large ("? epitheliall cells distended with fat globules. Specitic
gravity 1017. Diasnosis, distended gall bladder, chronic intlammatory induration.

In 1905 Alban Doran delivered an interesting clinical lecture at the Samaritan Free Hospital for Women upon Dilatation of the Gall Bladder Simulating Ovarian Cyst. I feel much indebted to that lecture, and have taken from it the following details, of which I would not otherwise have been arrare.

He describes his case of a gall bladder holding 2 pints of turbid fluid, together with a number of calculi. He then discusses the whole subject, dividing these conditions into three classes :

(a) Cystic tumours of great size extending to the left of the middle line (Terrier. Lawson 'Tait, Gersuny).

(b) Cystic tumours filling the greater part of the right side of the abdomen, liable to be taken for orarian cysts, fixed to that side by parictal adhesions (Kocher, Tuffellhis own casc).

(c) Relatively small dropsical gall bladders associated with a second tumour of more doubtful character (Raymond, Tischendorff, Chance).

For the purposes of my present paper it is only necessary to refer to those under class $(a)$, into which my case naturally falls:

1. Terrier's case readily heads the list by virtue of its truly enormous size. Woman, aged 50 years, from whose gall bladder 24 litres, or practically 42 pints, of gambogecoloured fluid was taken.

2. The case described in this paper falls into the next place from its very large si\%e. Woman, aged 31 years, from whose gall bladder twice there were 25 pints drawn off, and from whom at the opcration 22 pints of thick gamboge-colonred liquid were taken.

3. Lawson Tait's case. Woman aged 40 years. Eleren pints of glairy liquid taken out.

4. Gersuny's case. Woman aged 50 years. Quantity not given, but an " enormous quantity of bile."

Erdmann related a remarknble case of a man, aged 24 years, from whom $60 \mathrm{lb}$. to $80 \mathrm{lb}$. of bile.stained finid was removed by tapping. As no exploratory operation was performed, owin to his leaving the hospital, the relations of the cyst remain unknown. Alban Doran suggests that "it was most probably a cystic gall bladder, but that it niolit have developer inside the liver as a bile cyst, the gall bladder and ducts remaining normal." For the references to these cases quoted from the lecture by Alban Doran I would refer readers to the full report of it in the British Midic.sL JourNal of June 17th, 1905.

The mechanics of the development in my patient of such on enormous gall bladder with so thick a wall is interesting. Apparently in some way the fall led to an occlusion of the common duct, though the specitic cause is not evident. The absence of any calculi in the cyst rather negatives the probability of one or more causing the obstruction. It is to be regretted that the urgency of her bodily condition was so serious that careful examination of the liver and ducts was impossible. The tension of the bile in the hepatic and cystic ducts must have been con siderable to so greatly stretch the gall bladder and hypertrophy its walls. During this time her health suffered and jaundice was present. In consequence of the clrawing off of twenty.five pints the tension was reduced and the jaundice vanished although no bile was apparently entering the intestinal tract. After the first and second tappings the gall bladder from its overstretched condition and absence of contractile power served only as a reservoir. 
After the removal of the sac, gradually the sinus from the cystic duct contracted and at the same time the kinking, or other canse of the occlusion of the common duct, was remored and the bile passed into the intestine and continues to do so, as shown by the colour of the faoces.

The age of my patient was less than usually is the case where the gall bladder is much dilated. The rate of dilatation was much greater, as usually the process is a slow one. Twenty.five pints were drawn off within four or five weeks of any swelling being observed, and not more than seven months after the fall which most probably set up the condition.

REFERENCE.

1 Britisir Medical JodRsaL, June 17th, 1905, p. 1316.

\section{SECONDARY PARO'TITIS DUE TO ORAL STARVATION IN THE MEDICAL TREATMENT OF GASTRIC ILCER.}

H. D. ROLLESTON, and M.D., F.R.C.P., ST. GEORGE's HOSIITAL. SENIOR InYSICIIN,
M. W. B. OLIVER, M.R.C.S., L.R.C.P., HoVSF-PHYSICIAN,
T. GFORGE'S HOSPITAL.
THE occurrence of secondary or symptomatic parotitis is well recognized as a result of abdominal diseases, especially after laparotomy for perforated gastric ulcer and in cases obviously infected, such as appendicitis, in fevers, in gastric ulcer, and in some other conditions. Stephen Paget first drew attention to the parotitis secondary to abdominal lesions in 1886, and in the following year he collected 101 cases, 50 of which were associated with some morbid condition of the generative organs. In 1904 the etiology and pathology of secondary parotitis were fully discussed by Bucknall and by Tebbs. Bucknall brought forward histological evidence in support of the conclusion that parotitis is invariably due to an ascending infection of Stenson's duct depending on oral sepsis, and that its onset may be prevented by appropriate measures. Tebbs, on the other band, considered that in the majority of cases the path of infection is the blood stream, and not the duct from the mouth.

In the present communication we desire to direct special attention to the occurrence of secondary parotitis in the course of gastric ulcer treated medically by oral starvation so as to ensure absolute rest to the stomach. One of us (H. D. R.), after trials with other methods, such as Lenhartz's and the various moditications of oral feeding bclieves that the safest method as regards the ulcer, and the one most likely to be followed by permanent ctre, is the somewhat Spartan one of giving nothing, not even water, by the mouth, and supplying 3 or 4 pints daily of water or (in recent years) of saline solution con taining sugar, or of albumen water, by the rectum until deep tenderness over the stomach has disapp ared, nutrient enemas being given in special circumstances only. The reason for depriving the patient of water by the mouth, even in sips, is of course that, since it is not atsorbed by the stomach, its expulsion necessitates peristaltic action, and thus interferes with healing of the ulcer and, as has happencd in some cases, may give rise to haematemesis.

The drawbacks to this method of treatment are the discomfort due to thirst and the occasional occurrence of parotitis, to which we wish to draw special attention. During eleven years' work in the wards of St. George's Hospital one of us (H. D. R.) has employed this method of treatment in the great majority of acute gastric ulcers and of chronic gastric ulcers accompanied by haemorrhage; during this period secondary parotitis has supervened in 9 cases of gastric ulcer under his care. It may be mentioned that special attention has always been paid to the mouth in the way of ordering antiseptic month washes in these cases, so as to avoid this complication. Such an incidence of secondary parotitis in cases of gastric ulcer treated medically by oral starvation appears to be exceptional, if we may judge from the scanty referonces to this subject in medical literature and from conversations with some other hospital physicians. The largest published collection of cases is that given by Tebbs- namely, 15 cases among the cases of gastric ulcer treated medically in St. George's Hospital between the years 1890 and 1904 . These cases are included in our series of 23 cases collected from the notes of cases in the hospital during the rather longer period of the twenty. years 1889-1908. Paget's original paper in 1886, which. summarized 60 cases of secondary parotitis, contained. one case of acute gastric ulcer with suppurative parotitis, the patient surviving but never recovering her "healte, of mind or body." In 1898 Hone reported a fatal caseof suppurative parotitis in a patient treated by starvation for gastric ulcer, and collected 8 racorded cases-Hawthorne 2 cases, Donkin 3 cases, E. A. Barton 2 cases, Nicholson 1 case. In his textbook Osler mentions that he has seen 3 cases after gastric ulcer, but as no details are given we have not counted these as due solely tc. oral starvation. Campbell Howard states that in fifteen years at the Johns Hopkins Hospital there were 76 cases. of ulcer of the stomach with 1 fatal case of suppurative parotitis (previously recorded by Atkinson).

In the twenty years-1889 to 1908-there were 1,000 cases of gastric ulcer treated by medical measures in St. George's Hospital. As we are anxious to confine our investigation to the influence of oral starvation in the causation of secondary parotitis, we have not included any cases of gastric ulcer which were operated upon in the following statistics. Secondary parotitis has been shown to occur in a considerable proportion of cases of gastric ulcer after operation (English, Tebbs), and may then be associated with, and possibly related to, peritoneal infection. Of the 1,000 cases, 530 received food, water, or both, by the mouth from the outset of treatment, whilst the remaining 470 cases were not allowed anything by the mouth for some days after admission. Among the 1,00C. cases there were 23 cases of parotitis, or 2.3 per cent., alk in women; in no case was any patient affected twice with this complication. Of these 23 cases complete oral starvation was employed in 21 ; the two remaining cases were on rectal feeding but in addition were allowed to suck ice. In other words, the percentage of secondary parotitis in 470 cases treated by rigid oral starration was. 4.5, whilst in the 530 cases allowed something, even though only water, by the mouth it was 0.4 . Haematemesis had occurred, either before admission or while in the hospital, in 16 of the 23 cases of gastric ulcer complicated by parotitis. It is thus clear that exclusive rectal feeding. or. rather oral starvation, is a much more constant antecedent. than haematemesis. Attention is drawn to this point. because Hone attached more importance to antecedent haematemesis than to oral starvation as a factor in the causation of this form of parotitis. Probably haematemesis is likely to be a factor in the production of parotitis only in so far as it leads to treatment by oral starva. tion. There is no evidence that secondary parotitis depends on the occurrence in the parotid gland of throm-. bosis such as might be favoured by a post-haemorrhagic. leucocytosis.

Of the 23 cases, the parotitis was confined to the left. side in 9 cases, and in 2 cases the left side was affected first and the right side later; in 6 cases the right side only was affected, and in 1 case the right side was affected first and the left side subsequently; in 5 cases the side affected was not noted. On two occasions secondary parotitis occurred in two patients about the same time; but, apart from this, there was no evidence that it occurred in epidemic form. Of the 23 cases, suppuration occurred. in 4,2 of which were fatal; in one of these two the suppuration spread diffusely into the tissues of the neck, and was indistinguishable from Ludwig's angina. Suppura. tive parotitis in the course of gastric ulcer is a grave complication, for out of 8 cases of suppurative parotitis (4 from St. George's Hospital, and cases recorded by Paget, Hone, Hawthorne, and Barton) 3 proved fatal. Among. the remaining 19 patients of gastric ulcer, with non. suppurative parotitis, in St. George's Hospital, 2 died.

In conclusion, we have collected 34 cases of secondary parotitis in cases of gastric ulcer, 23 from St. George's Hospital and 11 pablished by others (Paget 1, Hone 9; Atkinson 1). From the consideration of the exceptional number observed at St. George's Hospital, an event which we cannot explain, it appears (1) that secondary parotitis may complicate cases of gastric ulcer treated medically by oral starvation; (2) that it occurs ten and a half times, 